Research Article

\title{
Toeplitz Type Operators Associated with Generalized Calderón-Zygmund Operator on Weighted Morrey Spaces
}

\author{
Bijun Ren and Enbin Zhang \\ Department of Information Engineering, Henan College of Finance and Taxation, Zhengzhou, Henan 451464, China \\ Correspondence should be addressed to Enbin Zhang; 13526812071@163.com
}

Received 10 February 2016; Accepted 27 March 2016

Academic Editor: Maria Alessandra Ragusa

Copyright (C) 2016 B. Ren and E. Zhang. This is an open access article distributed under the Creative Commons Attribution License, which permits unrestricted use, distribution, and reproduction in any medium, provided the original work is properly cited.

Let $T_{1}$ be a generalized Calderón-Zygmund operator or $\pm I$ (the identity operator), let $T_{2}$ and $T_{4}$ be the linear operators, and let $T_{3}= \pm I$. Denote the Toeplitz type operator by $T^{b}=T_{1} M^{b} I_{\alpha} T_{2}+T_{3} I_{\alpha} M^{b} T_{4}$, where $M^{b} f=b f$ and $I_{\alpha}$ is the fractional integral operator. In this paper, we investigate the boundedness of the operator $T^{b}$ on weighted Morrey space when $b$ belongs to the weighted BMO spaces.

\section{Introduction and Results}

The classical Morrey spaces, introduced by Morrey [1] in 1938, have been studied intensively by various authors and together with weighted Lebesgue spaces play an important role in the theory of partial differential equations (see [2, 3]). Komori and Shirai [4] introduced a version of the weighted Morrey space $L^{p, \kappa}(\omega)$, which is a natural generalization of the weighted Lebesgue space $L^{p}(\omega)$.

Definition 1. Suppose that $T: \mathcal{S} \rightarrow \mathcal{S}^{\prime}$ is a linear operator with kernel $K(\cdot, \cdot)$ defined initially by

$$
\begin{aligned}
T(f)(x)=\int_{\mathbb{R}^{n}} K(x, y) f(y) d y, & \\
& f \in C_{c}^{\infty}\left(\mathbb{R}^{n}\right), x \notin \operatorname{supp} f .
\end{aligned}
$$

The operator $T$ is called a generalized Calderón-Zygmund operator provided that the following three conditions are satisfied:

(1) $T$ can be extended into a continuous operator on $L^{2}\left(\mathbb{R}^{n}\right)$.
(2) $K$ is smooth away from the diagonal $\{(x, y): x=y\}$ with

$$
\begin{aligned}
& \int_{|x-y|>2|z-y|}(|K(x, y)-K(x, z)| \\
& +|K(y, x)-K(z, x)|) d x \leq C,
\end{aligned}
$$

where $C>0$ is a constant independent of $y$ and $z$.

(3) There is a sequence of positive constant numbers $\left\{C_{j}\right\}$ such that, for each $j \in N$,

$$
\begin{aligned}
& \left(\int_{2^{j}|z-y| \leq|x-y|<2^{j+1}|z-y|}|K(x, y)-K(x, z)|^{q} d x\right)^{1 / q} \\
& \quad \leq C_{j}\left(2^{j}|z-y|\right)^{-n / q^{\prime}},
\end{aligned}
$$

$$
\begin{aligned}
& \left(\int_{2^{j}|z-y| \leq|x-y|<2^{j+1}|z-y|}|K(y, x)-K(z, x)|^{q} d x\right)^{1 / q} \\
& \quad \leq C_{j}\left(2^{j}|z-y|\right)^{-n / q^{\prime}},
\end{aligned}
$$

where $\left(q, q^{\prime}\right)$ is a fixed pair of positive numbers with $1 / q+1 / q^{\prime}=1$ and $1<q^{\prime}<2$. 
If we compare the generalized Calderón-Zygmund operator with the classical Calderón-Zygmund operator, whose kernel $K$ is smooth away from the diagonal $\{(x, y): x=y\}$ with

$$
\begin{aligned}
& |K(x, y)| \leq C|x-y|^{-n}, \\
& |K(x, y)-K(x, z)|+|K(y, x)-K(z, x)| \\
& \quad \leq C|x-y|^{-n}\left(\frac{|z-y|}{|x-y|}\right)^{\delta},
\end{aligned}
$$

where $|x-y|>2|z-y|$ for some $\delta>0$, we can find out that the classical Calderón-Zygmund operator is a generalized Calderón-Zygmund operator defined above with $C_{j}=2^{-j \delta}$, $j \in N$, and any $1<q<\infty$.

Let $b$ be a locally integrable function on $\mathbb{R}^{n}$. The Toeplitz type operator associated with generalized CalderónZygmund operator and fractional integral operator $I_{\alpha}$ is defined by

$$
T^{b}=T_{1} M^{b} I_{\alpha} T_{2}+T_{3} I_{\alpha} M^{b} T_{4},
$$

where $T_{1}$ is the generalized Calderón-Zygmund operator or $\pm I$ (the identity operator), $T_{2}$ and $T_{4}$ are the linear operators, $T_{3}= \pm I$, and $M^{b} f=b f$.

Note that the commutators $\left[b, I_{\alpha}\right](f)=b I_{\alpha}(f)-I_{\alpha}(b f)$ are the particular cases of the Toeplitz type operators $T^{b}$. The Toeplitz type operators $T^{b}$ are the nontrivial generalization of these commutators.

The boundedness of the singular integral commutators generated by $\mathrm{BMO}$ function was obtained in [5-8]. Motivated by these, in this paper, we investigate the boundedness of $T^{b}$ on the weighted Morrey space when $b$ belongs to weighted $\mathrm{BMO}$ space and we have the following result.

Theorem 2. Suppose that $T^{b}$ is a Toeplitz type operator associated with generalized Calderón-Zygmund operator and fractional integral operator $I_{\alpha}$, and $b \in B M O(\omega)$. Let $0<\alpha<$ $n, q^{\prime}<p<n / \alpha, 1 / s=1 / p-\alpha / n, 0<\kappa<p / s,\left\{j C_{j}\right\} \in l^{1}$, $\omega^{s / p} \in A_{1}$ and the critical index of $\omega$ for the reverse Hölder condition $r_{\omega}>\max \left\{(s-1) q^{\prime} /\left(s-q^{\prime}\right),(1-\kappa) /(p / s-\kappa)\right\}$. If $T^{1}(f)=0$ for any $f \in L^{p, \kappa}(\omega), T_{2}$ and $T_{4}$ are the bounded operators on $L^{p, \kappa}(\omega)$, and then there exists a constant $C>0$ such that

$$
\left\|T^{b}(f)\right\|_{L^{s, \kappa s / p}\left(\omega^{1-(1-\alpha / n) s}, \omega\right)} \leq C\|b\|_{B M O(\omega)}\|f\|_{L^{p, \kappa}(\omega)} .
$$

The following results are immediately obtained from Theorem 2.

Corollary 3 (see [5]). Let $0<\alpha<n, 1<p<n / \alpha, 1 / q=$ $1 / p-\alpha / n, 0<\kappa<p / q$, and $\omega^{q / p} \in A_{1}$. Suppose that $b \in$ $B M O(\omega)$ and the critical index of $\omega$ for the reverse Hölder condition $r_{\omega}>(1-\kappa) /(p / q-\kappa)$; then, $\left[b, I_{\alpha}\right]$ is bounded from $L^{p, \kappa}(\omega)$ to $L^{q, \kappa q / p}\left(\omega^{1-(1-\alpha / n) q}, \omega\right)$.

Corollary 4. Suppose that $T^{b}$ is a Toeplitz type operator associated with generalized Calderón-Zygmund operator and fractional integral operator $I_{\alpha}$ and $b \in B M O\left(\mathbb{R}^{n}\right)$. Let $0<\alpha<$ $n, 1<p<n / \alpha, 1 / s=1 / p-\alpha / n, 0<\kappa<p / s$, and $\left\{j C_{j}\right\} \in l^{1}$. If $T^{1}(f)=0$ for any $f \in L^{p, \kappa}\left(\mathbb{R}^{n}\right), T^{k, 2}$ and $T^{k, 4}$ are the bounded operators on $L^{p, \kappa}\left(\mathbb{R}^{n}\right)$; then, there exists a constant $C>0$ such that

$$
\left\|T^{b}(f)\right\|_{L^{s, \kappa s} p\left(\mathbb{R}^{n}\right)} \leq C\|b\|_{B M O}\|f\|_{L^{p, \kappa}\left(\mathbb{R}^{n}\right)} .
$$

The paper is organized as follows. In Section 2, we will introduce some notation and definitions and recall some preliminary results. In Section 3, we give the sharp estimates for $T^{b}$. In Section 4, we will give the proof of Theorem 2.

\section{Some Preliminaries}

First, let us recall some notation and the definition of weight classes.

A weight $\omega$ is a nonnegative, locally integrable function on $\mathbb{R}^{n}$. Let $B=B_{r}\left(x_{0}\right)$ denote the ball with the center $x_{0}$ and radius $r$, and let $\lambda B=B_{\lambda r}\left(x_{0}\right)$ for any $\lambda>0$. For a given weight function $\omega$ and a measurable set $E$, we also denote the Lebesgue measure of $E$ by $|E|$ and set weighted measure $\omega(E)=\int_{E} \omega(x) d x$. For any given weight function $\omega$ on $\mathbb{R}^{n}$, $0<p<\infty$, denote by $L^{p}(\omega)$ the space of all function $f$ satisfying

$$
\|f\|_{L^{p}(\omega)}=\left(\int_{\mathbb{R}^{n}}|f(x)|^{p} \omega(x) d x\right)^{1 / p}<\infty .
$$

Definition 5 (see [9]). A weight $\omega$ is said to belong to the Muckenhoupt class $A_{p}$ for $1<p<\infty$, if there exists a constant $C$ such that

$$
\left(\frac{1}{|B|} \int_{B} \omega(x) d x\right)\left(\frac{1}{|B|} \int_{B} \omega(x)^{-1 /(p-1)} d x\right)^{p-1} \leq C
$$

for every ball $B$. The class $A_{1}$ is defined by replacing the above inequality with

$$
\frac{1}{|B|} \int_{B} \omega(y) d y \leq C \cdot \underset{x \in B}{\operatorname{ess} \inf } w(x) .
$$

When $p=\infty$, we defined $A_{\infty}=\cup_{1 \leq p<\infty} A_{p}$.

Definition 6 (see [10]). A weight function $\omega$ belongs to $A_{p, q}$ for $1<p<q<\infty$, if for every ball $B$ in $\mathbb{R}^{n}$, there exists a positive constant $C$ which is independent of $B$ such that

$$
\left(\frac{1}{|B|} \int_{B} \omega(y)^{-p^{\prime}} d y\right)^{1 / p^{\prime}}\left(\frac{1}{|B|} \int_{B} \omega(y)^{q} d y\right)^{1 / q} \leq C,
$$

where $p^{\prime}$ denotes the conjugate exponent of $p>1$; that is, $1 / p+1 / p^{\prime}=1$.

From the definition of $A_{p, q}$, we can get that

$$
\omega \in A_{p, q}, \quad \text { iff } \omega^{q} \in A_{1+q / p^{\prime}} .
$$


Definition 7 (see [11]). A weight function $\omega$ belongs to the reverse Hölder class $\mathrm{RH}_{u}$ if there exist two constants $u>1$ and $C>0$ such that the following reverse Hölder inequality

$$
\left(\frac{1}{|B|} \int_{B} \omega(x)^{u} d x\right)^{1 / u} \leq C\left(\frac{1}{|B|} \int_{B} \omega(x) d x\right)
$$

holds for every ball $B \subset \mathbb{R}^{n}$.

It is well known that if $\omega \in A_{p}$ with $1<p<\infty$, then $\omega \in A_{u}$ for all $u>p$ and $\omega \in A_{q}$ for some $1<q<p$. If $\omega \in A_{p}$ with $1 \leq p<\infty$, then there exists $u>1$ such that $\omega \in \mathrm{RH}_{u}$. It follows directly from Hölder's inequality that $\omega \in \mathrm{RH}_{u}$ implies $\omega \in \mathrm{RH}_{s}$ for all $1<s<u$. Moreover, if $\omega \in \mathrm{RH}_{u}, u>1$, then we have $\omega \in \mathrm{RH}_{u+\varepsilon}$ for some $\varepsilon>0$. We write $r_{\omega}=\sup \left\{u>1: \omega \in \mathrm{RH}_{u}\right\}$ to denote the critical index of $\omega$ for the reverse Hölder condition.

Lemma 8 (see [11]). Suppose $\omega \in A_{1}$. Then, there exist two constants $C_{1}$ and $C_{2}$, such that

$$
C_{1} \omega(B) \leq|B| \inf _{x \in B} \omega(x) \leq C_{2} \omega(B) .
$$

Lemma 9 (see [11]). Let $\omega \in A_{p}, p \geq 1$. Then, for any ball $B$ and any $\lambda>1$, there exists an absolute constant $C>0$ such that

$$
\omega(\lambda B) \leq C \lambda^{p n} \omega(B),
$$

where $C$ does not depend on $B$ or $\lambda$.

Next, we will recall the definition of the Hardy-Littlewood maximal operator and several variants, the fractional integral operator, and some function spaces.

Definition 10. The Hardy-Littlewood maximal operator $M f$ is defined by

$$
M(f)(x)=\sup _{x \in B} \frac{1}{|B|} \int_{B}|f(y)| d y .
$$

For $0<\delta<1$, the sharp maximal operator $M_{\delta}^{\sharp} f$ is defined by

$$
M_{\delta}^{\sharp}(f)(x)=\sup _{x \in B} \inf _{c \in \mathbb{C}}\left(\left.\frac{1}{|B|} \int_{B}|| f(y)\right|^{\delta}-|c|^{\delta} \mid d y\right)^{1 / \delta} .
$$

For $0 \leq \alpha<n, t \geq 1$, we define the fractional maximal operator $M_{\alpha, t} f$ by

$$
M_{\alpha, t}(f)(x)=\sup _{x \in B}\left(\frac{1}{|B|^{1-\alpha t / n}} \int_{B}|f(y)|^{t} d y\right)^{1 / t}
$$

and define the fractional weighted maximal operator $M_{\alpha, r, \omega} f$ by

$$
\begin{aligned}
& M_{\alpha, t, \omega} f(x) \\
& \quad=\sup _{x \in B}\left(\frac{1}{\omega(B)^{1-\alpha t / n}} \int_{B}|f(y)|^{t} \omega(y) d y\right)^{1 / t},
\end{aligned}
$$

where the above supremum is taken over all balls $B$ containing $x$. In order to simplify the notation, we set $M_{\alpha}=M_{\alpha, 1}$ and $M_{t, \omega}=M_{0, t, \omega}$.
Definition 11. For $0<\alpha<n$, the fractional integral operator $I_{\alpha}$ is defined by

$$
I_{\alpha}(f)(x)=\int_{\mathbb{R}^{n}} \frac{f(y)}{|x-y|^{n-\alpha}} d y .
$$

Lemma 12. Let $I_{\alpha}$ be fractional integral operator, and let $E$ be a measurable set in $\mathbb{R}^{n}$. Then, for any $f \in L^{1}\left(\mathbb{R}^{n}\right)$, there exists a constant $C$ such that

$$
\int_{E}\left|I_{\alpha} f(x)\right| d x \leq C\|f\|_{L^{1}}|E|^{\alpha / n} .
$$

Proof. Since

$$
\begin{aligned}
& \left|\left\{x \in E:\left|I_{\alpha} f(x)\right|>\lambda\right\}\right| \leq\left|\left\{x \in R^{n}:\left|I_{\alpha} f(x)\right|>\lambda\right\}\right| \\
& \quad \leq C\left(\frac{\|f\|_{L^{1}}}{\lambda}\right)^{n /(n-\alpha)},
\end{aligned}
$$

we have

$$
\begin{gathered}
\int_{E}\left|I_{\alpha} f(x)\right| d x=\int_{0}^{\infty}\left|\left\{x \in E:\left|I_{\alpha} f(x)\right|>\lambda\right\}\right| d \lambda \\
\leq \int_{0}^{\infty} \min \left\{C\left(\frac{\|f\|_{L^{1}}}{\lambda}\right)^{n /(n-\alpha)},|E|\right\} d \lambda \\
\leq \int_{0}^{C\|f\|_{L^{1}}|E|^{\alpha / n-1}}|E| d \lambda \\
\quad+\int_{C\|f\|_{L^{1}}|E|^{\alpha / n-1}}^{\infty}\left(\frac{\|f\|_{L^{1}}}{\lambda}\right)^{n /(n-\alpha)} d \lambda \\
\leq C\|f\|_{L^{1}}|E|^{\alpha / n} .
\end{gathered}
$$

Definition 13. Let $1 \leq p<\infty$ and $\omega$ be a weighted function. A locally integrable function $b$ is said to be in $\mathrm{BMO}_{p}(\omega)$ if

$$
\begin{aligned}
& \|b\|_{\mathrm{BMO}_{p}(\omega)} \\
& \quad=\sup _{B}\left[\frac{1}{\omega(B)} \int_{B}\left|b(x)-b_{B}\right|^{p} \omega(x)^{1-p} d x\right]^{1 / p} \\
& \quad<\infty
\end{aligned}
$$

where $b_{B}=(1 /|B|) \int_{B} b(y) d y$ and the supremum is taken over all balls $B \subset \mathbb{R}^{n}$.

Lemma 14 (see [12]). Let $\omega \in A_{1}$. Then, for any $1 \leq p<\infty$, there exists an absolute constant $C>0$ such that $\|b\|_{B M O_{p}(\omega)} \leq$ $C\|b\|_{B M O(\omega)}$.

Definition 15 (see [4]). Let $1 \leq p<\infty, 0<\kappa<1$, and let $\omega$ be a weight function. Then, the weighted Morrey space is defined by

$$
L^{p, \kappa}(\omega)=\left\{f \in L_{\mathrm{loc}}^{p}(\omega):\|f\|_{L^{p, \kappa}(\omega)}<\infty\right\},
$$


where

$$
\|f\|_{L^{p, \kappa}(\omega)}=\sup _{B}\left(\frac{1}{\omega(B)^{\kappa}} \int_{B}|f(x)|^{p} \omega(x) d x\right)^{1 / p},
$$

and the supremum is taken over all balls $B \subset \mathbb{R}^{n}$.

In order to deal with the fractional order case, we need to consider the weighted Morrey space with two weights.

Definition 16 (see [4]). Let $1 \leq p<\infty$ and $0<\kappa<1$. Then, for two weights $\mu$ and $v$, the weighted Morrey space is defined by

$$
L^{p, \kappa}(\mu, \nu)=\left\{f \in L_{\mathrm{loc}}^{p}(\mu):\|f\|_{L^{p, \kappa}(\mu, v)}<\infty\right\},
$$

where

$$
\|f\|_{L^{p, \kappa}(\mu, \nu)}=\sup _{B}\left(\frac{1}{\nu(B)^{\kappa}} \int_{B}|f(x)|^{p} \mu(x) d x\right)^{1 / p},
$$

and the supremum is taken over all balls $B \subset \mathbb{R}^{n}$.

We list a series of lemmas which will be used in the proof of our theorem.

Lemma 17 (see [5]). Let $0<\alpha<n, 1<p<n / \alpha, 1 / q=$ $1 / p-\alpha / n, 0<\kappa<p / q$, and $\omega \in A_{\infty}$. Then, for any $1 \leq r<p$, we have

$$
\left\|M_{\alpha, r, \omega}(f)\right\|_{L^{q, \kappa q / p}(\omega)} \leq C\|f\|_{L^{p, \kappa}(\omega)} .
$$

Lemma 18 (see [5]). Let $0<\alpha<n, 1<p<n / \alpha, 1 / q=$ $1 / p-\alpha / n, \omega^{p / q} \in A_{1}$, and $r_{\omega}>(1-\kappa) /(p / q-\kappa)$. Then, for every $0<\kappa<p / q$ and $1 \leq r<p$, we have

$$
\left\|M_{r, \omega}(f)\right\|_{L^{q, \alpha q / p}\left(\omega^{q / p}, \omega\right)} \leq C\|f\|_{L^{q, \kappa q / p}\left(\omega^{q / p}, \omega\right)} .
$$

Lemma 19 (see [5]). Let $0<\alpha<n, 1<p<n / \alpha, 1 / q=$ $1 / p-\alpha / n$, and $\omega^{p / q} \in A_{1}$. Then, if $0<\kappa<p / q$ and $r_{\omega}>$ $(1-\kappa) /(p / q-\kappa)$, we have

$$
\left\|M_{\alpha}(f)\right\|_{L^{q, \kappa q / p}\left(\omega^{q / p}, \omega\right)} \leq C\|f\|_{L^{p, \kappa}(\omega)} .
$$

Lemma 20 (see [4]). Let $0<\alpha<n, 1<p<n / \alpha, 1 / q=$ $1 / p-\alpha / n$, and $\omega^{p / q} \in A_{1}$. Then, if $0<\kappa<p / q$, we have

$$
\left\|I_{\alpha}(f)\right\|_{L^{q, \kappa q / p}\left(\omega^{q / p}, \omega\right)} \leq C\|f\|_{L^{p, \kappa}(\omega)} .
$$

\section{The Sharp Estimates for $T^{b}$}

In this section, we will prove the sharp estimates for $T^{b}$ as follows.

Theorem 21. Suppose that $T^{b}$ is a Toeplitz type operator associated with generalized Calderón-Zygmund operator and fractional integral operator $I_{\alpha}$, and $b \in B M O(\omega)$. Let $0<\delta<$ $1,0<\alpha<n, q^{\prime}<p<n / \alpha,\left\{j C_{j}\right\} \in l^{1}, \omega \in A_{1}, r_{\omega}>q^{\prime}$, and $t>\left(r_{\omega}-1\right) q^{\prime} /\left(r_{\omega}-q^{\prime}\right)$. If $T^{1}(f)=0$ for any $f \in L^{p, \kappa}(\omega)$, then there exists a constant $C>0$ such that

$$
\begin{aligned}
& M_{\delta}^{\sharp}\left(T^{b}(f)\right)(x) \leq C\|b\|_{B M O(\omega)} \omega(x) \\
& \quad \cdot\left(M_{t, \omega}\left(I_{\alpha} T_{2} f\right)(x)+M_{\alpha}\left(T_{4} f\right)(x)\right) \\
& \quad+C\|b\|_{B M O(\omega)} \omega(x)^{1-\alpha / n} M_{\alpha, t, \omega}\left(T_{4} f\right)(x) .
\end{aligned}
$$

Proof. For any ball $B=B\left(x_{0}, r_{B}\right)$ which contains $x$, without loss generality, we may assume that $T_{1}$ is a generalized Calderón-Zygmund operator. We write, by $T^{1}(f)=0$,

$$
\begin{aligned}
T^{b}(f)(y) & =T_{1} M^{b} I_{\alpha} T_{2}(f)(y)+T_{3} I_{\alpha} M^{b} T_{4}(f)(y) \\
& =\mathcal{U}^{b}(y)+\mathscr{V}^{b}(y) \\
& =\mathcal{U}^{b-b_{2 B}}(y)+\mathscr{V}^{b-b_{2 B}}(y),
\end{aligned}
$$

where

$$
\begin{aligned}
\mathcal{U}^{b-b_{2 B}}(y)= & T_{1} M^{\left(b-b_{2 B}\right) \chi_{2 B}} I_{\alpha} T_{2}(f)(y) \\
& +T_{1} M^{\left(b-b_{2 B}\right) \chi_{(2 B)}} I_{\alpha} T_{2}(f)(y) \\
= & U_{1}(y)+U_{2}(y), \\
\mathscr{V}^{b-b_{2 B}}(y)= & T_{3} I_{\alpha} M^{\left(b-b_{2 B}\right) \chi_{2 B}} T_{4}(f)(y) \\
& +T_{3} I_{\alpha} M^{\left(b-b_{2 B}\right) \chi_{(2 B)}} T_{4}(f)(y) \\
= & V_{1}(y)+V_{2}(y) .
\end{aligned}
$$

Since $0<\delta<1$, then

$$
\begin{aligned}
& \left(\left.\frac{1}{|B|} \int_{B}|| T^{b}(f)(y)\right|^{\delta}-\left|U_{2}\left(x_{0}\right)+V_{2}\left(x_{0}\right)\right|^{\delta} \mid d y\right)^{1 / \delta} \\
& \leq\left(\frac{1}{|B|} \int_{B}\left|T^{b}(f)(y)-U_{2}\left(x_{0}\right)-V_{2}\left(x_{0}\right)\right|^{\delta} d y\right)^{1 / \delta} \\
& \leq\left(\frac{1}{|B|} \int_{B}\left|U_{1}(y)\right|^{\delta} d y\right)^{1 / \delta} \\
& \quad+\left(\frac{1}{|B|} \int_{B}\left|V_{1}(y)\right|^{\delta} d y\right)^{1 / \delta} \\
& \quad+\left(\frac{1}{|B|} \int_{B}\left|U_{2}(y)-U_{2}\left(x_{0}\right)\right|^{\delta} d y\right)^{1 / \delta} \\
& \quad+\left(\frac{1}{|B|} \int_{B}\left|V_{2}(y)-V_{2}\left(x_{0}\right)\right|^{\delta} d y\right)^{1 / \delta} \\
& =M_{1}+M_{2}+M_{3}+M_{4} .
\end{aligned}
$$


We are going to estimate each term, respectively. Since $T_{1}$ is bounded from $L^{1}$ to $W L^{1}$ and $\omega \in A_{1}$, then, by Kolmogorov's inequality and Lemmas 8 and 9 , we get

$$
\begin{aligned}
M_{1} & =\left(\frac{1}{|B|} \int_{B}\left|U_{1}(y)\right|^{\delta} d y\right)^{1 / \delta} \leq \frac{1}{|B|} \\
\cdot & \int_{2 B}\left|\left(b(y)-b_{2 B}\right) I_{\alpha} T_{2}(f)(y)\right| d y \\
& \leq \frac{C}{|B|}\left(\int_{2 B}\left|b(y)-b_{2 B}\right|^{t^{\prime}} \omega(y)^{1-t^{\prime}} d y\right)^{1 / t^{\prime}} \\
& \cdot\left(\int_{2 B}\left|I_{\alpha} T_{2} f(y)\right|^{t} \omega(y) d y\right)^{1 / t} \leq C\|b\|_{\mathrm{BMO}(\omega)} \\
& \cdot \frac{\omega(2 B)}{|B|} M_{t, \omega}\left(I_{\alpha} T_{2} f\right)(x) \leq C\|b\|_{\mathrm{BMO}(\omega)} \omega(x) \\
\cdot & M_{t, \omega}\left(I_{\alpha} T_{2} f\right)(x) .
\end{aligned}
$$
have

Since $T_{3}= \pm I$, by Hölder's inequality and Lemma 12, we

$$
\begin{aligned}
M_{2} & =\left(\frac{1}{|B|} \int_{B}\left|T_{3} I_{\alpha} M^{\left(b-b_{2 B}\right) \chi_{2 B}} T_{4}(f)(y)\right|^{\delta} d y\right)^{1 / \delta} \\
& \leq \frac{C}{|B|} \int_{B}\left|T_{3} I_{\alpha} M^{\left(b-b_{2 B}\right) \chi_{2 B}} T_{4}(f)(y)\right| d y=\frac{C}{|B|} \\
& \cdot \int_{B}\left|I_{\alpha} M^{\left(b-b_{2 B}\right) \chi_{2 B}} T_{4}(f)(y)\right| d y \leq C \frac{1}{|B|^{1-\alpha / n}} \\
& \cdot \int_{\mathbb{R}^{n}}\left|M^{\left(b-b_{2 B}\right) \chi_{2 B}} T_{4}(f)(y)\right| d y \leq C \\
& \cdot \frac{1}{|B|^{1-\alpha / n}}\left(\int_{2 B}\left|b(y)-b_{2 B}\right|^{t^{\prime}} \omega(y)^{1-t^{\prime}} d y\right)^{1 / t^{\prime}} \\
& \cdot\left(\int_{2 B}\left|T_{4}(f)(y)\right|^{t} \omega(y) d y\right)^{1 / t} \leq C\|b\|_{\mathrm{BMO}(\omega)} \\
& \cdot\left(\frac{\omega(B)}{|B|}\right)^{1-\alpha / n} \\
& \cdot\left(\frac{1}{\omega(B)^{(1-\alpha t / n)}} \int_{2 B}\left|T_{4}(f)(y)\right|^{t} \omega(y) d y\right)^{1 / t} \\
& \leq C\|b\|_{\mathrm{BMO}(\omega)} \omega(x)^{1-\alpha / n} M_{\alpha, t, \omega}\left(T_{4} f\right)(x) .
\end{aligned}
$$

By the definition of generalized Calderón-Zygmund operator, we have

$$
\begin{aligned}
& \left|U_{2}(y)-U_{2}\left(x_{0}\right)\right|=\mid T_{1} M^{\left(b-b_{2 B}\right) \chi_{(2 B)}} I_{\alpha} T_{2}(f)(y) \\
& \quad-T_{1} M^{\left(b-b_{2 B}\right) \chi_{(2 B)}} I_{\alpha} T_{2}(f)\left(x_{0}\right) \mid \\
& \quad \leq C \int_{(2 B)^{c}}\left|b(z)-b_{2 B}\right|\left|K(y, z)-K\left(x_{0}, z\right)\right| \\
& \cdot\left|I_{\alpha} T_{2}(f)(z)\right| d z .
\end{aligned}
$$

Then, by Hölder's inequality, we get

$$
\begin{aligned}
& M_{3}=\left(\frac{1}{|B|} \int_{B}\left|U_{2}(y)-U_{2}\left(x_{0}\right)\right|^{\delta} d y\right)^{1 / \delta} \leq \frac{1}{|B|} \\
& \cdot \int_{B}\left|U_{2}(y)-U_{2}\left(x_{0}\right)\right| d y \leq \frac{1}{|B|} \\
& \cdot \int_{B} \mid T_{1} M^{\left(b-b_{2 B}\right) \chi_{(2 B)^{c}}} I_{\alpha} T_{2}(f)(y) \\
& -T_{1} M^{\left(b-b_{2 B}\right) \chi_{(2 B)}{ }^{c}} I_{\alpha} T_{2}(f)\left(x_{0}\right) \mid d y \leq \frac{C}{|B|} \\
& \cdot \int_{B} \int_{(2 B)^{c}}\left|b(z)-b_{2 B}\right|\left|K(y, z)-K\left(x_{0}, z\right)\right| \\
& \cdot\left|I_{\alpha} T_{2}(f)(z)\right| d z d y \leq \frac{C}{|B|} \\
& \cdot \sum_{j=1}^{\infty} \int_{B} \int_{2^{j}\left|y-x_{0}\right| \leq\left|z-x_{0}\right|<2^{j+1}\left|y-x_{0}\right|}\left|b(z)-b_{2^{j+1} B}\right| \\
& \cdot\left|K(y, z)-K\left(x_{0}, z\right)\right|\left|I_{\alpha} T_{2}(f)(z)\right| d z d y \\
& +\frac{C}{|B|} \sum_{j=1}^{\infty}\left|b_{2^{j+1} B}-b_{2 B}\right| \\
& . \\
& \quad \int_{B} \int_{2^{j}\left|y-x_{0}\right| \leq\left|z-x_{0}\right|<2^{j+1}\left|y-x_{0}\right|}\left|K(y, z)-K\left(x_{0}, z\right)\right| \\
& \cdot\left|I_{\alpha} T_{2}(f)(z)\right| d z d y=M_{31}+M_{32} .
\end{aligned}
$$

Since $r_{\omega}>q^{\prime}>1$ and $t>\left(r_{\omega}-1\right) q^{\prime} /\left(r_{\omega}-q^{\prime}\right)>q^{\prime}$, then there exists $1<l<\infty$ such that $1 / q+1 / t+1 / l=1$. Applying Hölder's inequality for $q, l$, and $t$ and by (3) of Definition 1 , we get

$$
\begin{aligned}
& M_{31} \leq \frac{C}{|B|} \\
& \cdot \sum_{j=1}^{\infty} \int_{B}\left(\int_{2^{j}\left|y-x_{0}\right| \leq\left|z-x_{0}\right|<2^{j+1}\left|y-x_{0}\right|}\left|K(y, z)-K\left(x_{0}, z\right)\right|^{q} d z\right)^{1 / q} \\
& \cdot\left(\int_{2^{j}\left|y-x_{0}\right| \leq\left|z-x_{0}\right| 2^{j+1}\left|y-x_{0}\right|}\left|b(z)-b_{2^{j+1} B}\right|^{l} \omega(z)^{\left(1 / t^{\prime}-1\right) l} d z\right)^{1 / l} \\
& \cdot\left(\int_{2^{j}\left|y-x_{0}\right| \leq\left|z-x_{0}\right| 2^{j+1}\left|y-x_{0}\right|}\left|I_{\alpha} T_{2}(f)(z)\right|^{t} \omega(z) d z\right)^{1 / t} d y \\
& \leq \frac{C}{|B|} \sum_{j=1}^{\infty} C_{j} \int_{B}\left(2^{j}\left|y-x_{0}\right|\right)^{-n / q^{\prime}} d y \\
& \cdot\left(\int_{2^{j+1} B}\left|b(z)-b_{2^{j+1} B}\right|^{l} \omega(z)^{\left(1 / t^{\prime}-1\right) l} d z\right)^{1 / l} \\
& \cdot\left(\int_{2^{j+1} B}\left|I_{\alpha} T_{2}(f)(z)\right|^{t} \omega(z) d z\right)^{1 / t} .
\end{aligned}
$$

Note that

$$
\int_{B}\left(2^{j}\left|y-x_{0}\right|\right)^{-n / q^{\prime}} d y \leq C\left|2^{j} B\right|^{-1 / q^{\prime}} r^{n}
$$


then,

$$
\begin{gathered}
M_{31} \leq C M_{t, \omega}\left(I_{\alpha} T_{2} f\right)(x) \sum_{j=1}^{\infty} C_{j}\left|2^{j} B\right|^{-1 / q^{\prime}} \omega\left(2^{j} B\right)^{1 / t} \\
\cdot\left(\int_{2^{j+1} B}\left|b(z)-b_{2^{j+1} B}\right|^{l} \omega(z)^{\left(1 / t^{\prime}-1\right) l} d z\right)^{1 / l} \cdot
\end{gathered}
$$

Since $t>\left(r_{\omega}-1\right) q^{\prime} /\left(r_{\omega}-q^{\prime}\right)$, we have $r_{\omega}>(t-1) q^{\prime} /\left(t-q^{\prime}\right)$. By $r_{\omega}=\sup \left\{u>1: \omega \in \mathrm{RH}_{u}\right\}$, there is $u$ such that $u>$ $(t-1) q^{\prime} /\left(t-q^{\prime}\right)>q^{\prime}>1$. Let $p_{0}=(u-1) /\left((t-1) q^{\prime} /(t-\right.$ $\left.\left.q^{\prime}\right)-1\right)$; then, $1<p_{0}<\infty$. By $1 / q+1 / l+1 / t=1$, we have $l / t^{\prime}=(t-1) q^{\prime} /\left(t-q^{\prime}\right)$. Then, $p_{0}=(u-1) /\left(l / t^{\prime}-1\right)$; that is, $u=l p_{0} / t^{\prime}-p_{0} / p_{0}^{\prime}$. Then, we have

$$
\begin{aligned}
& \frac{(1-u)}{\left(p_{0} l\right)}-\frac{1}{q^{\prime}}=-1, \\
& \frac{1}{t}+\frac{1}{\left(p_{0}^{\prime} l\right)}+\frac{u}{\left(p_{0} l\right)} .
\end{aligned}
$$

Applying Hölder's inequality for $p_{0}$ and $p_{0}^{\prime}$, the definition of weighted $\mathrm{BMO}$, and $\omega \in \mathrm{RH}_{u}$, we have

$$
\begin{aligned}
& \left(\int_{2^{j+1} B}\left|b(z)-b_{2^{j+1} B}\right|^{l} \omega(z)^{\left(1 / t^{\prime}-1\right) l} d z\right)^{1 / l} \\
& \quad \leq C\left(\int_{2^{j+1} B}\left|b(z)-b_{2^{j+1} B}\right|^{l p_{0}^{\prime}} \omega(z)^{1-l p_{0}^{\prime}} d z\right)^{1 /\left(p_{0}^{\prime} l\right)} \\
& \cdot\left(\int_{2^{j+1} B} \omega(z)^{u} d z\right)^{1 /\left(p_{0}^{\prime} l\right)} \leq C\|b\|_{\mathrm{BMO}(\omega)} \\
& \cdot\left|2^{j} B\right|^{(1-u) /\left(p_{0} l\right)} \omega\left(2^{j} B\right)^{1 /\left(p_{0}^{\prime} l\right)+u /\left(p_{0} l\right)} .
\end{aligned}
$$

Hence,

$$
\begin{aligned}
& M_{31} \leq C\|b\|_{\mathrm{BMO}(\omega)} M_{t, \omega}\left(I_{\alpha} T_{2} f\right)(x) \\
& \cdot \sum_{j=1}^{\infty} C_{j}\left|2^{j} B\right|^{(1-u) /\left(p_{0} l\right)-1 / q^{\prime}} \omega\left(2^{j} B\right)^{1 / t+1 /\left(p_{0}^{\prime} l\right)+u /\left(p_{0} l\right)} \\
& \leq C\|b\|_{\mathrm{BMO}(\omega)} M_{t, \omega}\left(I_{\alpha} T_{2} f\right)(x) \sum_{j=1}^{\infty} C_{j} \frac{\omega\left(2^{j} B\right)}{\left|2^{j} B\right|} \\
& \quad \leq C \omega(x)\|b\|_{\mathrm{BMO}(\omega)} M_{t, \omega}\left(I_{\alpha} T_{2} f\right)(x) .
\end{aligned}
$$

Note that

$$
\begin{aligned}
\left|b_{2^{j+1} B}-b_{2 B}\right| & \leq \sum_{k=1}^{j} \frac{1}{\left|2^{k} B\right|} \int_{2^{k+1} B}\left|b(z)-b_{2^{k+1} B}\right| d z \\
& \leq C_{j} \omega(x)\|b\|_{\mathrm{BMO}(\omega)} .
\end{aligned}
$$

Thus, by Hölder's inequality, we get

$$
\begin{aligned}
& M_{32} \leq \frac{C}{|B|} \\
& \cdot \sum_{j=1}^{\infty} \int_{B}\left(\int_{2^{j}\left|y-x_{0}\right| \leq\left|z-x_{0}\right|<2^{j+1}\left|y-x_{0}\right|}\left|K(y, z)-K\left(x_{0}, z\right)\right|^{q} d z\right)^{1 / q} \\
& \cdot\left|b_{2^{j+1} B}-b_{2 B}\right|\left|2^{j+1} B\right|^{1 / l}\left(\int_{2^{j+1} B}\left|I_{\alpha} T_{2}(f)(z)\right|^{t} d z\right)^{1 / t} d y \\
& \leq \frac{C}{|B|} \omega(x)\|b\|_{\mathrm{BMO}(\omega)} \sum_{j=1}^{\infty} j C_{j} \int_{B}\left(2^{j}\left|y-x_{0}\right|\right)^{-n / q^{\prime}} d y \\
& \cdot\left(\frac{1}{\omega\left(2^{j+1} B\right)} \int_{2^{j+1} B}\left|I_{\alpha} T_{2}(f)(z)\right|^{t} \omega(z) d z\right)^{1 / t}\left|2^{j+1} B\right|^{1 / l+1 / t} \\
& \leq\left.\left. C \omega(x)\|b\|_{\mathrm{BMO}(\omega)} M_{t, \omega}\left(I_{\alpha} T_{2} f\right)(x) \sum_{j=1}^{\infty} j C_{j}\right|^{j+1} B\right|^{1 / l+1 / t-1 / q^{\prime}} \\
& \quad \leq C \omega(x)\|b\|_{\mathrm{BMO}(\omega)} M_{t, \omega}\left(I_{\alpha} T_{2} f\right)(x) \sum_{j=1}^{\infty} j C_{j} \leq C \omega(x) \\
& \cdot\|b\|_{\mathrm{BMO}(\omega)} M_{t, \omega}\left(I_{\alpha} T_{2} f\right)(x) .
\end{aligned}
$$

Then,

$$
M_{3} \leq C \omega(x)\|b\|_{\mathrm{BMO}(\omega)} M_{t, \omega}\left(I_{\alpha} T_{2} f\right)(x) .
$$
Then,

For any $y \in B$ and $z \in(2 B)^{c}$, we have $|y-z| \sim\left|x_{0}-z\right|$.

$$
\begin{aligned}
M_{4} & =\left(\frac{1}{|B|} \int_{B}\left|V_{2}(y)-V_{2}\left(x_{0}\right)\right|^{\delta} d y\right)^{1 / \delta} \leq \frac{1}{|B|} \\
\cdot & \int_{B}\left|V_{2}(y)-V_{2}\left(x_{0}\right)\right| d y \leq \frac{1}{|B|} \\
\cdot & \int_{B} \mid T_{3} I_{\alpha} M^{\left(b-b_{2 B}\right) \chi_{(2 B)^{c}}} T_{4}(f)(y) \\
& -T_{3} I_{\alpha} M^{\left(b-b_{2 B}\right) \chi_{(2 B)^{c}}} T_{4}(f)\left(x_{0}\right) \mid d y \leq C \frac{1}{|B|} \\
\cdot & \int_{B} \int_{(2 B)^{c}}\left|b(z)-b_{2 B}\right|\left|\frac{1}{|y-z|^{n-\alpha}}-\frac{1}{\left|x_{0}-z\right|^{n-\alpha}}\right| \\
\cdot & \left|T_{4}(f)(z)\right| d z d y \leq C \frac{1}{|B|} \\
\cdot & \int_{B} \int_{(2 B)^{c}} \mid b(z)-b_{2 B} \frac{\left|x_{0}-y\right|}{\left|x_{0}-z\right|^{n-\alpha+1} \mid} \\
\cdot & \left|T_{4}(f)(z)\right| d z d y \\
& \leq C \sum_{j=1}^{\infty} \frac{r_{B}\left(2^{j} r_{B}\right)^{n-\alpha+1}}{\left|T_{2}(f)(z)\right| d z \leq C \sum_{j=1}^{\infty+1} B} 2^{-j}\left|b_{2^{j+1} B}-b_{2 B}\right| \\
& \left|b(z)-b_{2 B}\right|
\end{aligned}
$$




$$
\begin{aligned}
& \cdot \frac{1}{\left|2^{j+1} B\right|^{1-\alpha / n}} \int_{2^{j+1} B}\left|T_{4}(f)(z)\right| d z+C \sum_{j=1}^{\infty} 2^{-j} \\
& \cdot \frac{1}{\left|2^{j+1} B\right|^{1-\alpha / n}} \int_{2^{j+1} B}\left|b(z)-b_{2^{j+1} B}\right| \\
& \cdot\left|T_{4}(f)(z)\right| d z=M_{41}+N_{42} .
\end{aligned}
$$

Note that

$$
\left|b_{2^{j+1} B}-b_{2 B}\right| \leq C_{j}\|b\|_{\mathrm{BMO}(\omega)}
$$

then,

$$
\begin{aligned}
& M_{41}=C \sum_{j=1}^{\infty} 2^{-j}\left|b_{2^{j+1} B}-b_{2 B}\right| \frac{1}{\left|2^{j+1} B\right|^{1-\alpha / n}} \\
& \cdot \int_{2^{j+1} B}\left|T_{4}(f)(z)\right| d z \leq C\|b\|_{\mathrm{BMO}(\omega)} \omega(x) \\
& \cdot M_{\alpha}\left(T_{4} f\right)(x) \sum_{j=1}^{\infty} j 2^{-j} \leq C\|b\|_{\mathrm{BMO}(\omega)} \omega(x) \\
& \cdot M_{\alpha}\left(T_{4} f\right)(x) .
\end{aligned}
$$

By Hölder’s inequality,

$$
\begin{aligned}
& M_{42}=C \sum_{j=1}^{\infty} 2^{-j} \frac{1}{\left|2^{j+1} B\right|^{1-\alpha / n}} \int_{2^{j+1} B}\left|b(z)-b_{2^{j+1} B}\right| \\
& \cdot\left|T_{4}(f)(z)\right| d z \leq C \sum_{j=1}^{\infty} 2^{-j} \\
& \cdot \frac{1}{\left|2^{j+1} B\right|^{1-\alpha / n}}\left(\int_{2^{j+1} B}\left|b(z)-b_{2^{j+1} B}\right|^{t^{\prime}}\right. \\
& \left.\cdot \omega(z)^{1-t^{\prime}} d z\right)^{1 / t^{\prime}}\left(\int_{2^{j+1} B}\left|T_{4}(f)(z)\right|^{t}\right. \\
& \cdot \omega(z) d z)^{1 / t} \leq C\|b\|_{\mathrm{BMO}(\omega)} \\
& \cdot \sum_{j=1}^{\infty} 2^{-j}\left(\frac{\omega\left(2^{j+1} B\right)}{\left|2^{j+1} B\right|}\right)^{1-\alpha / n}\left(\frac{1}{\omega\left(2^{j+1} B\right)^{1-\alpha t / n}}\right. \\
& \left.\cdot \int_{2^{j+1} B}\left|T_{4}(f)(z)\right|^{t} \omega(z) d z\right)^{1 / t} \leq C\|b\|_{\mathrm{BMO}(\omega)} \\
& \cdot \omega(x)^{1-\alpha / n} M_{\alpha, t, \omega}\left(T_{4} f\right)(x) .
\end{aligned}
$$

Then,

$$
\begin{aligned}
M_{4} & \leq C\|b\|_{\mathrm{BMO}(\omega)}\left(\omega(x) M_{\alpha}\left(T_{4} f\right)(x)\right. \\
& \left.+\omega(x)^{1-\alpha / n} M_{\alpha, t, \omega}\left(T_{4} f\right)(x)\right) .
\end{aligned}
$$

Combining the estimates for $M_{1}, M_{2}, M_{3}$, and $M_{4}$, the proof of Theorem 21 is completed.

\section{Proof of Theorem 2}

To prove Theorem 2, we need the following analogy of the classical Fefferman-Stein inequality for the sharp maximal function $M_{\delta}^{\sharp} f$; its proof can be found in [13].

Lemma 22. Let $0<\delta<1,0<\kappa<1$, and $1<p<\infty$. If $\mu, v \in A_{\infty}$, then we have

$$
\left\|M_{\delta}(f)\right\|_{L^{p, \kappa}(\mu, \nu)} \leq C\left\|M_{\delta}^{\sharp} f\right\|_{L^{p, \kappa}(\mu, \nu)}
$$

for all functions $f$ such that the left hand side is finite.

Proof. It follows from $r_{\omega}>(s-1) q^{\prime} /\left(s-q^{\prime}\right)$ that $s>\left(r_{\omega}-\right.$ 1) $q^{\prime} /\left(r_{\omega}-q^{\prime}\right)$; then, there exists $t$ such that $s>t>\left(r_{\omega}-\right.$ 1) $q^{\prime} /\left(r_{\omega}-q^{\prime}\right)$. Note that

$$
s+1-\left(1-\frac{\alpha}{n}\right) s=\frac{s}{p}
$$

then, by Lemma 22 and Theorem 21, we have

$$
\begin{aligned}
& \left\|T^{b}(f)\right\|_{L^{s, \kappa s / p}\left(\omega^{1-(1-\alpha / n) s}, \omega\right)} \\
& \quad \leq\left\|M_{\delta}^{\sharp}\left(T^{b}(f)\right)\right\|_{L^{s, \kappa s / p}\left(\omega^{1-(1-\alpha / n) s}, \omega\right)} \leq C\|b\|_{\mathrm{BMO}(\omega)} \\
& \cdot\left(\left\|M_{t, \omega}\left(I_{\alpha} T_{2} f\right)\right\|_{L^{s, \kappa s / p}\left(\omega^{s / p}, \omega\right)}\right. \\
& \left.\quad+\left\|M_{\alpha}\left(T_{4} f\right)\right\|_{L^{s, \kappa s / p}\left(\omega^{s / p}, \omega\right)}\right)+C\|b\|_{\mathrm{BMO}(\omega)} \\
& \cdot\left\|M_{\alpha, t, \omega}\left(T_{4} f\right)\right\|_{L^{s, \kappa s / p}(\omega)} \cdot
\end{aligned}
$$

Since $r_{\omega}>(1-\kappa) /(p / s-\kappa)$, by Lemmas $17-20$, we get

$$
\begin{aligned}
& \left\|T^{b}(f)\right\|_{L^{s, s / p}\left(\omega^{1-(1-\alpha / n) s, \omega)}\right.} \\
& \quad \leq C\|b\|_{\mathrm{BMO}(\omega)}\left(\left\|I_{\alpha} T_{2} f\right\|_{L^{s, \kappa s} p\left(\omega^{s / p}, \omega\right)}+\left\|T_{4} f\right\|_{L^{p, \kappa}(\omega)}\right) \\
& \quad \leq C\|b\|_{\mathrm{BMO}(\omega)}\|f\|_{L^{p, \kappa}(\omega)} .
\end{aligned}
$$

This finishes the proof of Theorem 2.

\section{Competing Interests}

The authors declare that there are no competing interests regarding the publication of this paper.

\section{References}

[1] C. B. Morrey, "On the solutions of quasi-linear elliptic partial differential equations," Transactions of the American Mathematical Society, vol. 43, no. 1, pp. 126-166, 1938.

[2] D. K. Palagachev and L. G. Softova, "Singular integral operators, Morrey spaces and fine regularity of solutions to PDE's," Potential Analysis, vol. 20, no. 3, pp. 237-263, 2004.

[3] G. Di Fazio and M. A. Ragusa, "Interior estimates in Morrey spaces for strong solutions to nondivergence form equations with discontinuous coefficients," Journal of Functional Analysis, vol. 112, no. 2, pp. 241-256, 1993. 
[4] Y. Komori and S. Shirai, "Weighted Morrey spaces and a singular integral operator," Mathematische Nachrichten, vol. 282, no. 2, pp. 219-231, 2009.

[5] H. Wang, "Some estimates for commutators of fractional integral operators on weighted Morrey spaces," Acta Mathematica Sinica (Chinese Series), vol. 56, no. 6, pp. 889-906, 2013.

[6] M. A. Ragusa, "Homogeneous Herz spaces and regularity results," Nonlinear Analysis. Theory, Methods \& Applications. An International Multidisciplinary Journal. Series A: Theory and Methods, vol. 71, no. 12, pp. e1909-e1914, 2009.

[7] M. A. Ragusa, "Necessary and sufficient condition for a VMO function," Applied Mathematics and Computation, vol. 218, no. 24, pp. 11952-11958, 2012.

[8] M. A. Ragusa, "Cauchy-Dirichlet problem associated to divergence form parabolic equations," Communications in Contemporary Mathematics, vol. 6, no. 3, pp. 377-393, 2004.

[9] B. Muckenhoupt, "Weighted norm inequalities for the Hardy maximal function," Transactions of the American Mathematical Society, vol. 165, pp. 207-226, 1972.

[10] B. Muckenhoupt and R. Wheeden, "Weighted norm inequalities for fractional integrals," Transactions of the American Mathematical Society, vol. 192, pp. 261-274, 1974.

[11] J. García-Cuerva and J. L. Rubio de Francia, Weighted Norm Inequalities and Related Topics, North-Holland, Amsterdam, The Netherlands, 1985.

[12] J. Garcia-Cuerva, "Weighted $H^{p}$ spaces," Dissertationes Mathematicae, vol. 162, pp. 1-63, 1979.

[13] P. Z. Xie and G. F. Cao, "Toeplitz-type operators in weighted Morrey spaces," Journal of Inequalities and Applications, vol. 2013, article 253, 2013. 


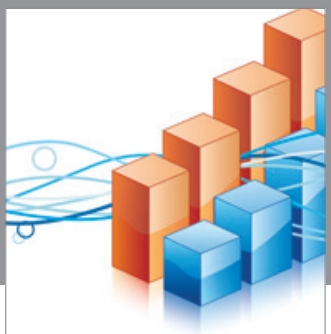

Advances in

Operations Research

vatem alat4

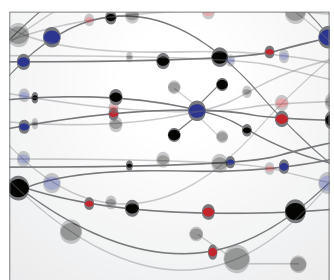

\section{The Scientific} World Journal
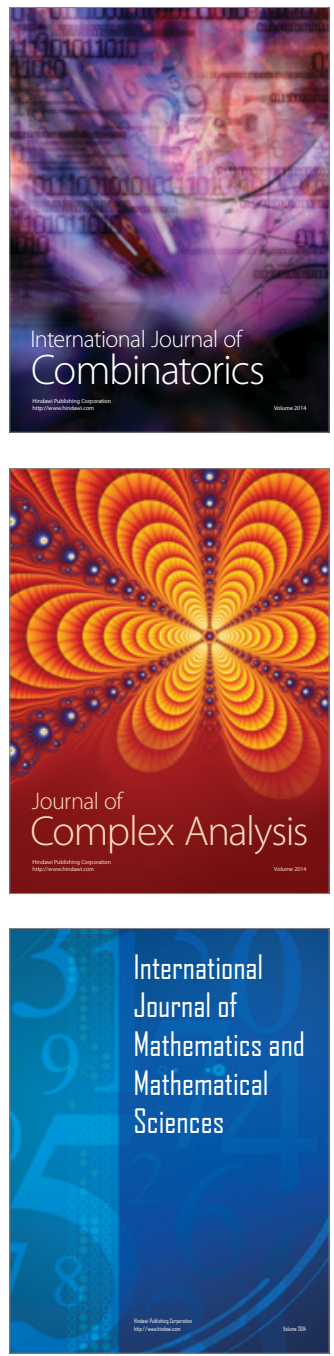
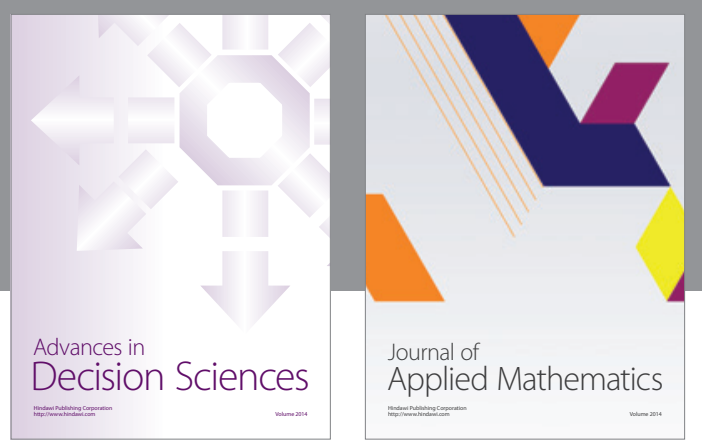

Algebra

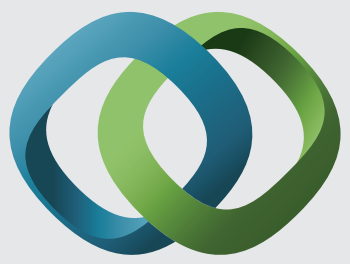

\section{Hindawi}

Submit your manuscripts at

http://www.hindawi.com
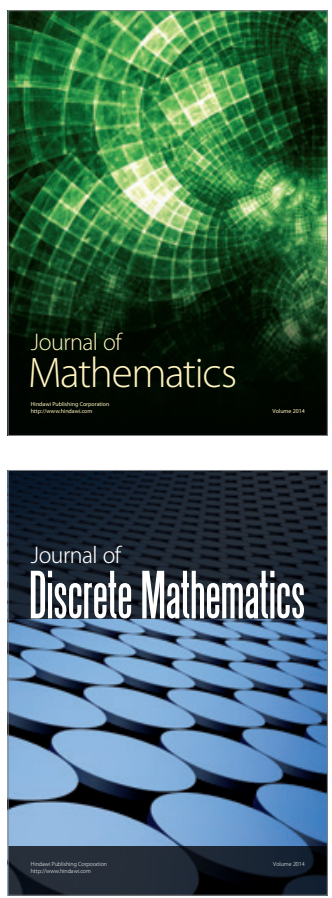

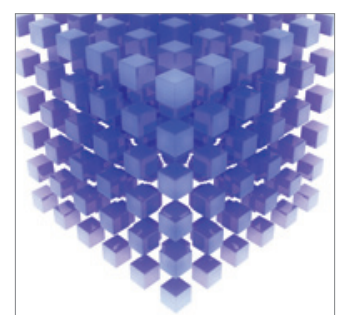

Mathematical Problems in Engineering
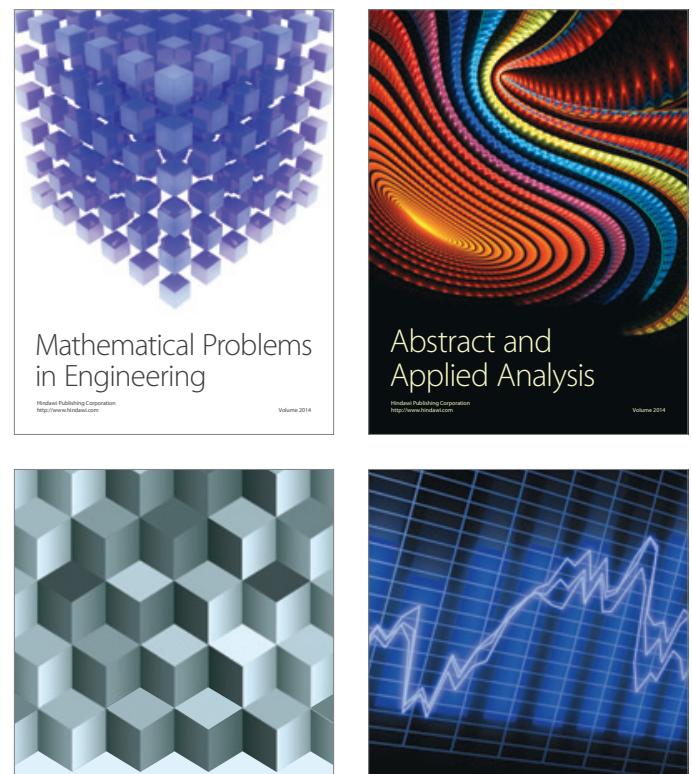

Journal of

Function Spaces

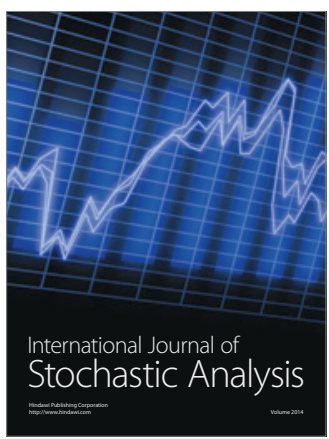

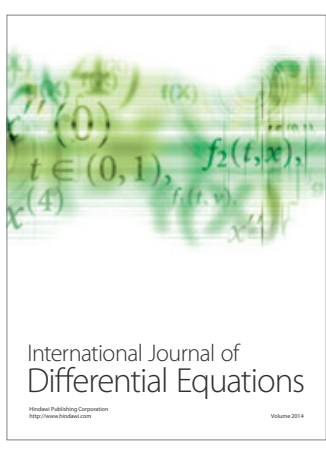
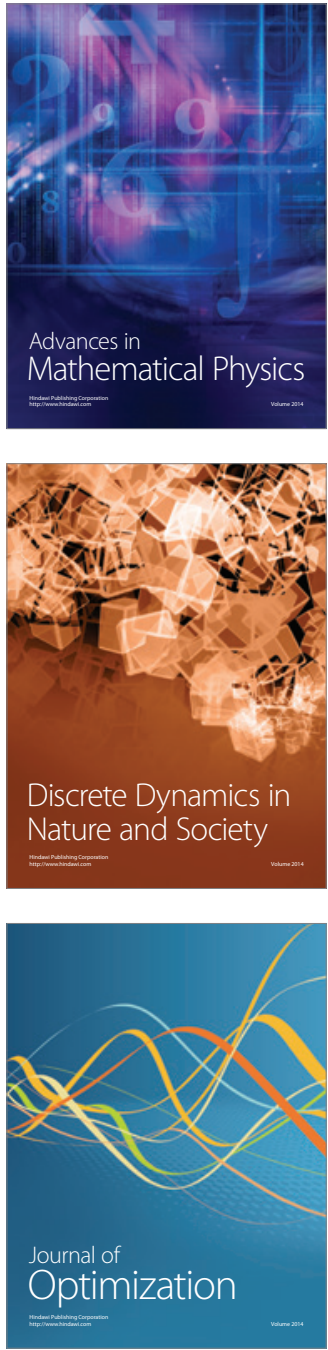